\title{
Enhancement of Angiogenesis by Ultrasound-Targeted Microbubble Destruction Combined with Nuclear Localization Signaling Peptides in Canine Myocardial Infarction
}

\author{
Jingjing Cui, ${ }^{1,2}$ Qing Deng, ${ }^{1}$ Qing Zhou, ${ }^{1}$ Sheng Cao, ${ }^{1}$ Nan Jiang, ${ }^{1}$ Yijia Wang, \\ Jinling Chen, ${ }^{1}$ Bo Hu, ${ }^{1}$ and Tuantuan Tan ${ }^{1}$ \\ ${ }^{1}$ Department of Ultrasound Imaging, Renmin Hospital of Wuhan University, Wuhan 430000, China \\ ${ }^{2}$ Department of Ultrasound Imaging, Affliated Hospital of Jining Medical University, Jining 272000, China
}

Correspondence should be addressed to Qing Zhou; qingzhou.wh.edu@hotmail.com

Received 23 April 2017; Revised 23 August 2017; Accepted 7 September 2017; Published 12 November 2017

Academic Editor: Magali Cucchiarini

Copyright ( 2017 Jingjing Cui et al. This is an open access article distributed under the Creative Commons Attribution License, which permits unrestricted use, distribution, and reproduction in any medium, provided the original work is properly cited.

Objective. This study aimed to develop a gene delivery system using ultrasound-targeted microbubbles destruction (UTMD) combined with nuclear localization signal (NLS) and investigate its efficacy and safety for therapeutic angiogenesis in canine myocardial infarction (MI) model. Methods. Fifty MI dogs were randomly divided into 5 groups and transfected with Ang-1 gene plasmid: (i) group A: only injection of microbubbles and Ang-1 plasmid; (ii) group B: only UTMD mediated gene transfection; (iii) group C: UTMD combined with classical NLS mediated gene transfection; (iv) group D: UTMD combined with mutational NLS mediated transfection; and (v) group E: UTMD combined with classical NLS in the presence of a nucleus transport blocker. The mRNA and protein expression of Ang-1 gene, microvessel density (MVD) cardiac troponin I (cTnI), and cardiac function were determined after transfection. Results. The expression of mRNA and protein of Ang-1 gene in group $\mathrm{C}$ was significantly higher than that of the other groups (all $P<0.01$ ). The MVD of group $\mathrm{C}$ was 10.2-fold of group A and 8.1-fold of group E $(P<0.01)$. The cardiac function in group $\mathrm{C}$ was significant improvement without cTnI rising. Conclusions. The gene delivery system composed of UTMD and NLS is efficient and safe.

\section{Introduction}

Since the first gene therapy trial was launched at the clinical center of the National Institute for Health (NIH) using a retrovirus for treating severe combined immunodeficiency disease [1], gene therapy has been considered a prospective tool for difficult-to-treat diseases by using the clinical techniques that are currently available [2]. Ultrasound-targeted microbubble destruction (UTMD), which is a new nonviral vector system capable of increasing transmembrane transport through sonoporation, has been shown to provide targeted delivery of reporter genes, therapeutic genes, and antisense oligonucleotides to tissues and organs in a safe manner $[3,4]$. However, the potential applications for this method are still a challenge due to the poor transfection efficiency of the exogenously transferred genes in vivo $[5,6]$.
Poor transfer of DNA fragments from the cytoplasm to the nucleus is one of the major reasons for the low exogenous gene transfection efficiency [7, 8]. Labat-Moleur et al. [9] showed that only 1 plasmid DNA fragment per 100 fragments could enter the nucleus from the cytoplasm in the gene transfer with lipopolyamines. It has been acknowledged that the outcomes of gene therapy are highly associated with the delivery of transferred DNA to the nucleus. However, previous studies of therapeutic gene delivery using UTMD have paid more attention to increasing the penetration of the cell membrane via physical bioeffects $[10,11]$, and few studies have been carried out to target gene breakthrough in the nuclear barrier during UTMD transfection in an effective manner.

It is well known that exogenous DNA can be transferred into the nucleus via active transport facilitated by the nuclear 
localization signal (NLS) $[12,13]$. Active transport is an energy-consuming process. For the mechanism of UTMD, microbubbles may serve as cavitation nuclei to accomplish sonoporation during ultrasound irradiation which creates transient and reversible pores on the cell membrane. A large amount of energy was released during the process which could provide the energy needed for nuclear transport of NLS theoretically [14]. Therefore, it would be possible to develop a nucleophilic gene transfection system based on UTMD and NLS.

Angiogenin-1 (Ang-1) gene encodes the angiopoietin 1 , which is involved in the specific chemiotaxis of the endothelial cells in vivo and thereby mediates the migration of endothelial cells to injury sites [15]. Further, the gene contributes to elevating the binding affinity of both endothelial cells and the basement membrane, which consequently induce de novo capillary recruitment and decrease the permeability of the capillaries. Based these factors, this study aimed to develop a novel gene transfection system composed of UTMD and NLS and to investigate the therapeutic potential of this system for treating ischemic heart disease by transfecting the Ang-1 gene into a canine myocardial infarction model.

\section{Materials and Methods}

2.1. Animal Model of Myocardial Infarction. Male adult hybrid dogs (weighing $17.3 \pm 3.2 \mathrm{~kg}$ ) were supplied by the Animal Experimental Center of Wuhan University (Approval number SYXK2004-0027, Wuhan, China). The study protocols were approved by the Ethical Committee of Renmin Hospital of Wuhan University. The experiments were performed according to the Guide to the Care and Use of Experimental Animals of US National Institutes of Health.

The dog myocardial infarction (MI) model was induced using interventional embolization. Briefly, the dogs in a fasting state were anesthetized with pentobarbital sodium $(30 \mathrm{mg} / \mathrm{kg})$ followed by a tracheal cannula. After femoral artery puncture, a $6 \mathrm{~F}$ sheathing canal was inserted, followed by an infusion of heparin $(1000 \mathrm{U})$. Then, a $5 \mathrm{~F}$ catheter was inserted into the ostium of the left coronary artery, followed by confirmation using DSA (Philips FD20, Andover, MA, USA). Subsequently, the microguide wire and catheter were inserted through the $5 \mathrm{~F}$ catheter until the orifice of the second diagonal branch of left anterior descending artery (LAD) was reached. Then, gelfoam granules $(250 \mu \mathrm{m}$, Alicon, Hangzhou, China) were infused into the distal part of the second diagonal branch of LAD to block the blood circulation. MI was confirmed by an elevation of ST segments in the ECG.

2.2. Experimental Group. The animals with successful MI induction were randomly divided into 5 groups with 10 in each group.

Group A. It is control, given an intravenous injection of $1 \mathrm{ml}$ of SonoVue microbubbles (Bracco, Geneva, Switzerland), $1 \mathrm{ml}$ of Ang-1-EGFP plasmid (0.5 mg/ml; Gene Chem, Shanghai, China), and $1 \mathrm{ml}$ of saline.

Group B. It is ultrasound-mediated plasmid DNA transfection group, which was treated with a $1 \mathrm{ml}$ suspension of the SonoVue microbubbles, $1 \mathrm{ml}$ of Ang-1-EGFP plasmid, and $1 \mathrm{ml}$ of saline.

Group C. It is ultrasound combined with classic nuclear localization signal- (cNLS-) mediated plasmid DNA transfection, which was subjected to a suspension of $1 \mathrm{ml}$ of SonoVue microbubbles, $1 \mathrm{ml}$ ofAng-1-EGFP plasmid, and $1 \mathrm{ml}$ of cNLS (PKKKRKV; 0.67 mg/ml; Shanghai Science Peptide BioTech, Shanghai, China).

Group D. It is ultrasound combined with mutational nuclear localization signal- (mNLS-) mediated plasmid DNA transfection, which was treated with a mixture of $1 \mathrm{ml}$ of SonoVue microbubbles, $1 \mathrm{ml}$ of Ang-1-EGFP plasmid, and $1 \mathrm{ml}$ of mNLS (PKTKRKV; $0.67 \mathrm{mg} / \mathrm{ml}$; Shanghai Science Peptide BioTech, Shanghai, China) for transfection.

Group E. It is ultrasound combined with cNLS-mediated plasmid DNA transfection while adding wheat germ agglutinin (WGA, which is a nucleus transport blocker, Sigma-Aldrich, CA, USA). The transfection suspension consisted of $1 \mathrm{ml}$ of SonoVue microbubbles, $1 \mathrm{ml}$ of Ang-1-EGFP plasmid, $1 \mathrm{ml}$ of cNLS, and $0.3 \mathrm{ml}$ of WGA $(0.1 \mathrm{mg} / \mathrm{ml})$.

In this study, a SonoVue microbubble suspension was prepared according to the manufacturer's instruction manual. The microbubbles were filled with sulfur hexafluoride gas and encapsulated by a thin and flexible phospholipid monolayer. The average diameter of the microbubbles was approximately $2.5 \mu \mathrm{m}$, and the concentration was $2 \times 10^{8}-$ $5 \times 10^{8} / \mathrm{ml}$. The Ang-1-EGFP plasmid was constructed by GeneChem Technology Co., Ltd. (Shanghai, China). In this plasmid, the coding region of Ang-1 was cloned in the vector GV230, under the control of the human cytomegalovirus promoter/enhancer followed by the enhanced green fluorescent protein (EGFP), for visualization of protein expression. The constructed plasmid was transformed into E. coli strain DH5a for amplification and purified using the Wizard ${ }^{\mathrm{TM}}$ Maxiprep DNA Purification System (Promega Corporation, Madison, WI, USA). The NLS peptide was purified by highperformance liquid chromatography, and the optimal molar ratio of NLS and Ang-1-EGFP plasmid was $10^{4}: 1$, according to our previous experiments [16]. The suspension of NLS and Ang-1-EGFP plasmid was incubated at room temperature for 30 minutes and added to $1 \mathrm{ml}$ of SonoVue microbubble suspension. The mixture was incubated for $2 \mathrm{hrs}$ at room temperature, and the upper layer suspension of microbubbles attached to plasmid and NLS was used for intravenous injection.

2.3. Gene Transfection. Gene transfection was carried out $24 \mathrm{hrs}$ after MI via intravenous injection of $3 \mathrm{ml}$ of various suspensions according to the above group setting. UTMD was 
performed using a Vivid Q ultrasound diagnostic platform (GE Healthcare, WI, USA) equipped with a M5S transducer $(1.7-3.3 \mathrm{MHz})$. The parasternal short axis view at the level of papillary muscles was selected for ultrasound irradiation. The ultrasound exposure started right after microbubble injection, and it terminated when the intramyocardium microbubbles completely vanished. The mechanical index was set at 1.3 , and the gain was modulated to the maximal level during ultrasound irradiation.

Three days after transfection, three dogs from each group were sacrificed to collect the myocardium in the peripheral infarction area for the freezing section, real-time PCR, and Western Blot analysis. Twenty-eight days after transfection, seven dogs from each group were sacrificed to obtain the myocardium for the evaluation of the area of MI, Masson staining, and immunofluorescent analyses.

\subsection{Freezing Section Analysis. Frozen sections were prepared} based on the myocardium stored at $-80^{\circ} \mathrm{C}$ to detect the expression of Ang-1-EGFP plasmid. The sections were then observed under a BX51 fluorescence microscope (Olympus, Tokyo, Japan) to evaluate the expression of EGFP-labeled Ang-1 plasmid. The excitation wavelength was set at $488 \mathrm{~nm}$. The tests were performed in triplicate.

2.5. Real-Time PCR. Total RNA was extracted from the myocardium (100 mg) using Trizol (Invitrogen, CA, USA) according to the manufacturer's instructions. The cDNA synthesis was carried out with approximately $2 \mu \mathrm{g}$ of RNA using the PrimeScript ${ }^{\mathrm{TM}} \mathrm{RT}$ reagent Kit with gDNA Eraser (Takara, Dalian, China). Real-time PCR was conducted using SYBR Green with the primers $\left(5^{\prime}\right.$-GAAGGAAACCGAGCCTATTCAC- $3^{\prime}$ and $5^{\prime}$-CCACAAGCATCAAACCACCA- $3^{\prime}$ ) synthesized by Invitrogen (Shanghai, China) according to the previous study [16]. The mRNA level was normalized by GAPDH. PCR reactions were performed in a total volume of $10 \mu \mathrm{l}$, containing $5 \mu \mathrm{l}$ of $2 \mathrm{x}$ SYBR Premix, $0.2 \mu \mathrm{l}$ of each specific primer to a final concentration of $200 \mu \mathrm{l}$, and $1 \mu \mathrm{l}$ of cDNA template. The PCR conditions consisted of denaturation at $95^{\circ} \mathrm{C}$ for 1 minute, followed by 40 cycles of denaturation at $95^{\circ} \mathrm{C}$ for $15 \mathrm{~s}$, annealing at $58^{\circ} \mathrm{C}$ for $20 \mathrm{~s}$ and extension at $72^{\circ} \mathrm{C}$ for $45 \mathrm{~s}$. The amplification results for realtime PCR were calculated as $2(-\Delta \Delta \mathrm{Ct})$ [17].

2.6. Western Blot Analysis. The myocardium $(100 \mathrm{mg})$ was homogenized in RIPA lysis buffer containing protease and phosphatase inhibitors. Proteins were separated by electrophoresis on an SDS-PAGE gel and transferred to a PVDF membrane. The membrane was blocked in 5\% nonfat milk and incubated with anti-Ang-1 antibody (1:1000; ab133425; Abcam, Cambridge, MA, USA) overnight at $4^{\circ} \mathrm{C}$; then it was incubated with the HRP-conjugated IgG $(1: 2000$; ab97057; Abcam, Cambridge, MA, USA) for $1 \mathrm{~h}$ at room temperature. The protein band was evaluated using the ECL chemiluminescence system (KeyuShenlan Scientific Co., Ltd., Beijing, China). The housekeeping protein (GAPDH) was used as the internal standard for the Ang-1 calculation. The ratio of protein/GAPDH was used to quantify the Ang-1 protein levels in each group.

2.7. Microvascular Density. The density of microvessels was determined using immunohistochemistry for alpha smooth muscle actin $(\alpha$-SMA). To estimate microvascular density, capillaries and arterioles were counted in five fields per slide using fluorescence microscopy (200x; BX52, Olympus, Japan), and the average value was taken.

2.8. Cardiac Function Evaluation. Two-dimensional echocardiography was performed before transfection (baseline) and 28 days after transfection. The echo instrument was GE Vivid $\mathrm{Q}$, as mentioned earlier. The left ventricular end-diastolic dimension (LVEDD) and left ventricular end-systolic dimension (LVESD) were measured, and the left ventricular enddiastolic volume (LVEDV) and left ventricular end-systolic volume (LVESV) as well as the left ventricular ejection fraction (LVEF) were obtained by biplane Simpson's method.

2.9. Determination of Myocardial Fibrosis Degree and Infarction Size. Masson staining was performed to evaluate myocardial fibrosis. The normal myocardial fiber is shown in red, and the collagen fiber was shown in blue to distinguish normal myocardium from fibrosis tissue.

Regarding the evaluation of the infarct area, the excised heart was washed with normal saline, followed by removal of the atrium and auricle of the heart as well as the right ventricle. From the apex to the basal level, the left ventricle was transversely cut into five sections with an average thickness of $0.8-1.0 \mathrm{~cm}$. The myocardial tissue at the level of the papillary muscle for each group was photographed using a digital camera; then the images were analyzed using Image-ProPlus 6.0 software (Media Cybernetics, MD, USA). Areas in a white color were defined as the myocardial infarct zone. The ratio of the infarct area to the total area of the left ventricular at the papillary muscle level was calculated to assess the size of infarction after treatment.

2.10. Serum cTnI Determination. To determine the safety of the gene delivery system composed of UTMD and NLS, the level of myocardial enzyme cardiac Troponin I (cTnI) before and after transfection of each group was detected. Blood samples were collected from the ulnar vein at the time points before MI and $4 \mathrm{hrs}, 24 \mathrm{hrs}$ after MI (pre-MI, MI- $4 \mathrm{~h}, \mathrm{MI}-24 \mathrm{~h}$ ), and 1 day and 6 days after transfection (Trans-D1, Trans-D6), respectively. The serum cTnI was detected with the commercial kit using the Beckman Coulter Immunochemiluminescent system.

\section{Statistical Analysis}

The SPSS 20.0 and GraphPad 6.01 software packages were used for the data analysis. Measurement data were presented as the mean \pm standard deviation. The K-S test was used to determine the normal distribution of the data. ANOVA was used to compare multiple groups, and the LSD method was 


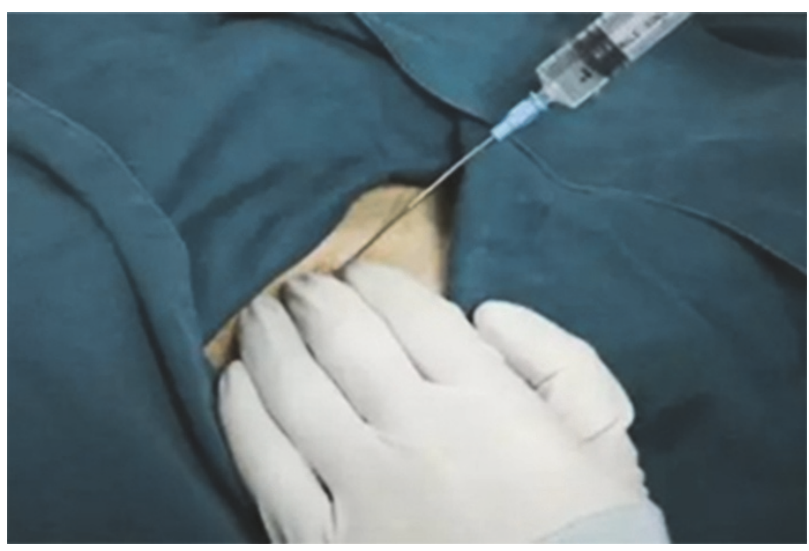

(a)

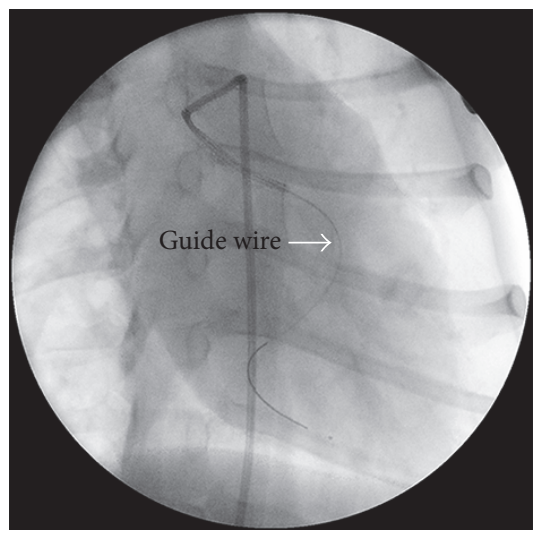

(c)

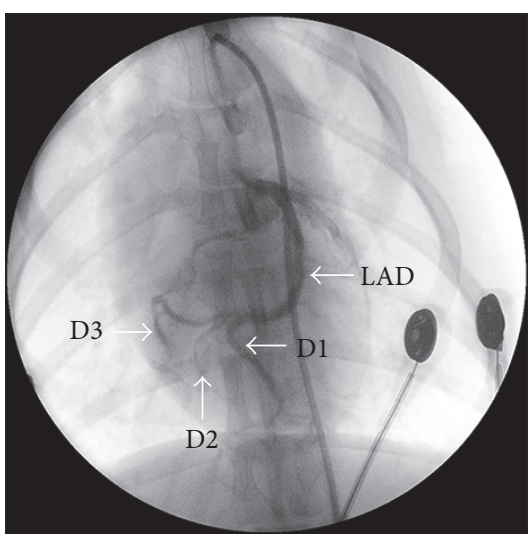

(d)

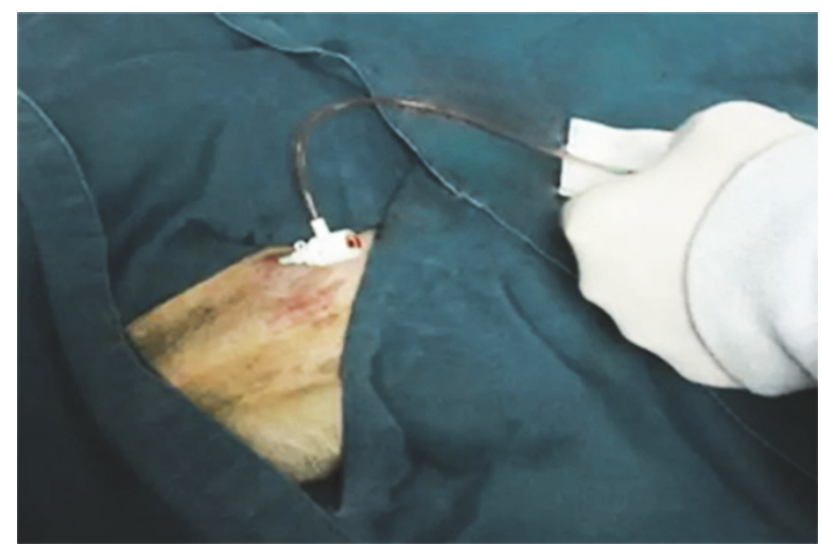

(b)

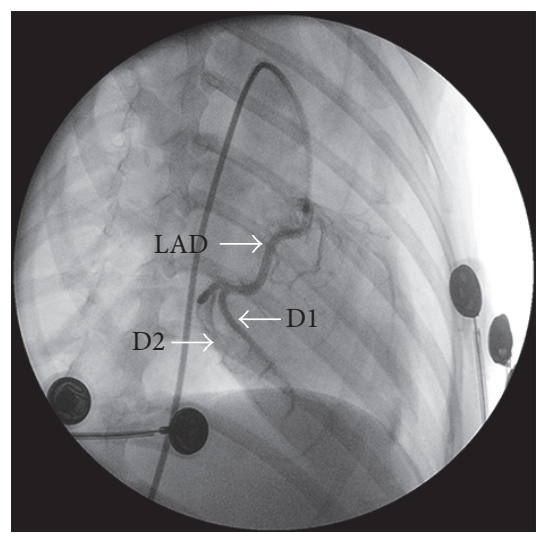

(e)

FIGURE 1: Schematic diagram of the myocardial infarction (MI) model using coronary artery embolism. (a) The puncture was performed at the obvious pulse location on the right femoral artery. (b) The artery sheath was inserted after puncture. (c) After the angiographic catheter reached the opening of the left coronary artery, the microguide wire and catheter could access the left anterior descending coronary artery. (d) The left coronary artery branches were clearly displayed before the MI modeling by coronary angiography. D1 = the first diagonal branch; D2 = the second diagonal branch; and D3 = the third diagonal branch. (e) After embolism of the coronary artery, the second diagonal branch distal of the left anterior descending artery was blocked. The second coronary angiography confirmed ischemia in the embolism area.

used for comparisons between two groups. $P<0.05$ was considered statistically significant.

\section{Results}

4.1. Mortality of Animal Model. Fifty-eight dogs received MI induction, among which two died from an anesthetic accident, three died from ventricular fibrillation, and three died died from operation. Finally, fifty dogs were confirmed with MI featured by elevation in the ST segment and blood circulation blockage on coronary angiography (Figure 1).

4.2. Plasmid Expression. Frozen section analysis showed EGFP was expressed in all five groups. The expression of EGFP was gradually increased from group A to group $\mathrm{C}$ and decreased from group $\mathrm{C}$ to group $\mathrm{E}$. The expression of EGFP peaked in group C (Figure 2).

Real-time PCR and Western Blot all showed that the expression of Ang-1 gene and protein were consistent with the expression of green fluorescent protein. For groups A, B,
C, D, and E, the relative expressions of Ang- 1 mRNA were $(1.0 \pm 0.01),(2.36 \pm 0.33),(4.25 \pm 0.43),(2.44 \pm 0.42)$, and $(1.43 \pm 0.23)$, respectively, and the relative expressions of Ang1 protein were $(0.15 \pm 0.06),(0.61 \pm 0.10),(0.99 \pm 0.13)$, $(0.59 \pm 0.10)$, and $(0.16 \pm 0.07)$, respectively. The relative expression of both Ang-1 mRNA and protein in group C was higher than in the other groups, with statistical significance $(P<0.05$, all, Figure 3$)$. The relative expression of Ang-1 protein in group $C$ was 1.6-fold higher than group $B$, which indicated that UTMD combined with NLS could effectively promote gene expression than UTMD alone (Figure 4). The closely equivalent results of groups B and D showed that the mutated NLS did not promote gene expression due to its loss of capacity for nuclear transport. The comparison of groups $\mathrm{C}$ and $\mathrm{E}$ indicated that the addition of WGA blocked nuclear transport and significantly decreased the expression of Ang-1 protein.

4.3. Microvascular Density. The newly formed capillary vessels were stained a red color by immunohistochemistry for $\alpha$ SMA. The density of the microvessels of group A, B, C, D, and 


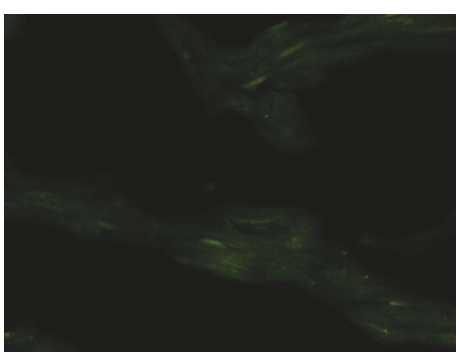

(a)

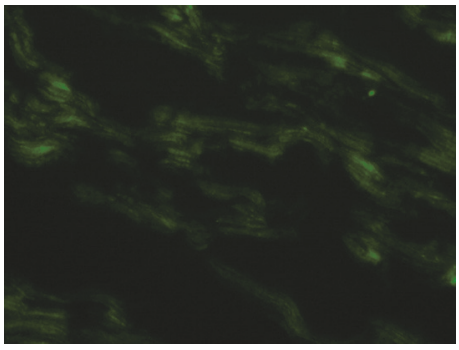

(b)

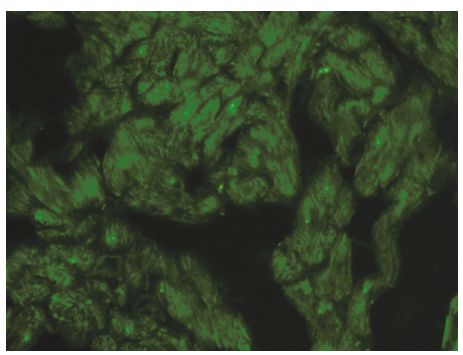

(c)

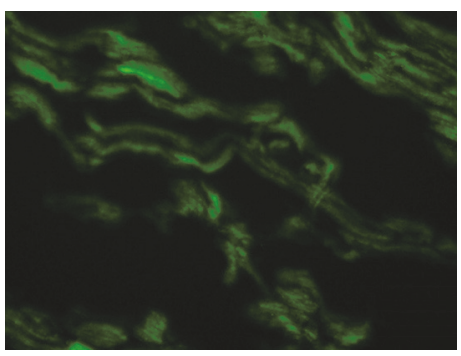

(d)

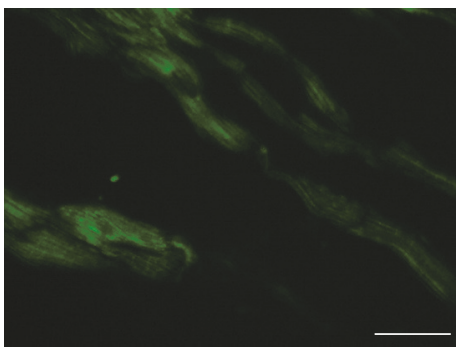

(e)

FIGURE 2: Representative micrographs for the expression of enhanced green fluorescent (EGFP) protein. EGFP in the border regions of $\mathrm{MI}$ was detected by confocal laser scanning microscopy on day 28 after transfection. The images were observed under a magnification of 400x. Scale bar $=50 \mu \mathrm{m}$. (a), (b), (c), (d), and (e) represented groups $\mathrm{A}, \mathrm{B}, \mathrm{C}, \mathrm{D}$ and $\mathrm{E}$, respectively.

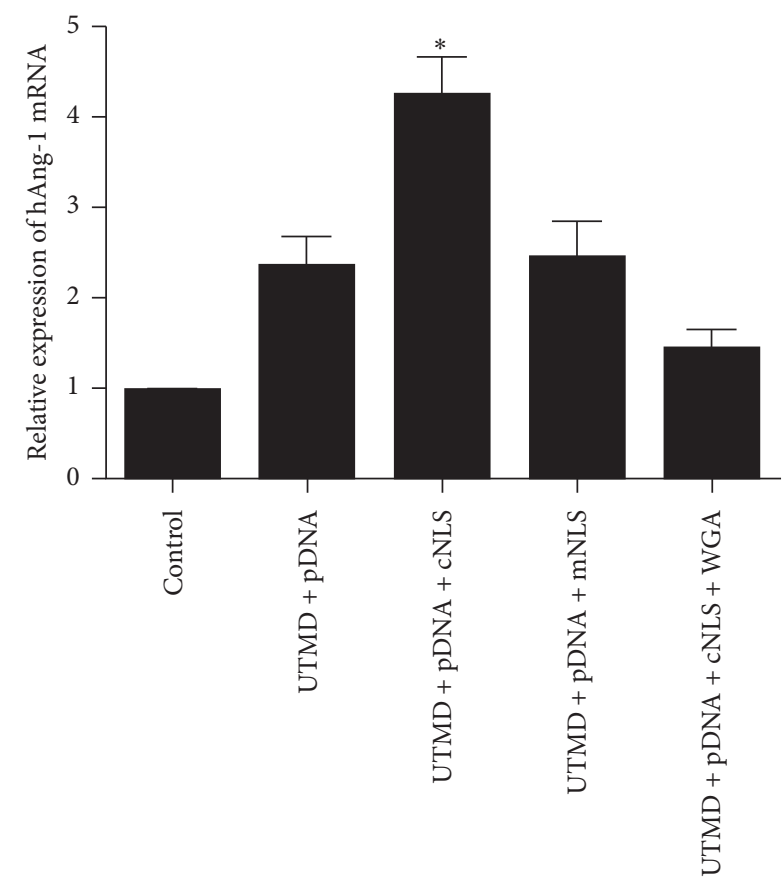

FIgure 3: Real-time PCR detects Ang-1 mRNA expression in the MI regions of canines 3 days after transfection. The Ang- 1 mRNA was normalized by GAPDH. The Ang-1 mRNA was higher in group C compared to the other groups, ${ }^{*} P<0.05$ compared to the other groups.

E was $\left(8.8 \pm 2.8 / \mathrm{mm}^{2}\right),\left(69.0 \pm 3.7 / \mathrm{mm}^{2}\right),\left(89.3 \pm 6.1 / \mathrm{mm}^{2}\right)$, $\left(71.3 \pm 11.6 / \mathrm{mm}^{2}\right)$, and $\left(11.0 \pm 4.3 / \mathrm{mm}^{2}\right)$, respectively. The microvascular density in group $\mathrm{C}$ was statistically higher than that in the other four groups (Figure 5).

4.4. Cardiac Function Improvement. No significant differences were noticed in the dimension or volume of the left ventricle or in LVEF among all five groups $(P>0.05)$ before transfection. The LVEF of groups A, B, C, D, and E at day 28 after gene transfection was $(29.7 \pm 5.8 \%),(41.6 \pm 2.6 \%)$, $(46.9 \pm 2.7 \%),(40.4 \pm 2.6 \%)$, and $(33.6 \pm 2.0 \%)$, respectively. Compared to the LV systolic function at baseline, the LVEF of groups $\mathrm{A}$ and $\mathrm{E}$ was further decreased, whereas that of groups $B$ and D was slightly increased. The LVEF of group $C$ showed a significant improvement compared to the other groups after transfection $(P<0.01$, Table 1$)$.

4.5. Myocardial Fibrosis and Infarct Size Assessment. Masson staining revealed that the degree of myocardial fibrosis in the five groups decreased from $\mathrm{A}$ to $\mathrm{C}$, and groups $\mathrm{D}$ and $\mathrm{E}$ were more severe than group $\mathrm{C}$. The myocardial fibrosis in group $\mathrm{C}$ was the least serious among the five groups (Figure 6).

Similarly, the MI area in group $\mathrm{C}$ was the lowest among the five groups (Figure 7). The infarct areas of groups A, B, C, D, and E accounted for approximately $1 / 2,1 / 4,1 / 5,1 / 4$, and $1 / 3$ of the left ventricular area, respectively. These results indicated that UTMD combined with NLS-mediated Ang-1 gene delivery contributed to the attenuation of the myocardial fibrosis and MI area. 
TABLE 1: Cardiac function in each group 28 days after treatment $(\bar{x} \pm s)$.

\begin{tabular}{lcccccccccc}
\hline \multirow{2}{*}{ Groups } & \multicolumn{2}{c}{ LVEDD $(\mathrm{mm})$} & \multicolumn{2}{c}{ LVESD $(\mathrm{mm})$} & \multicolumn{2}{c}{ LVEDV $(\mathrm{ml})$} & \multicolumn{2}{c}{ LVESV $(\mathrm{ml})$} & \multicolumn{2}{c}{ LVEF $(\%)$} \\
& Baseline & $28 \mathrm{~d}$ & Baseline & $28 \mathrm{~d}$ & Baseline & $28 \mathrm{~d}$ & Baseline & $28 \mathrm{~d}$ & Baseline & $28 \mathrm{~d}$ \\
\hline Group A & $27.1 \pm 3.1^{\mathrm{ce}}$ & $29.3 \pm 2.9^{\mathrm{c}}$ & $22.4 \pm 2.5$ & $25.3 \pm 1.7$ & $29.6 \pm 9.4$ & $33.2 \pm 8.0^{\mathrm{c}}$ & $18.4 \pm 5.7$ & $23.1 \pm 4.1$ & $38.0 \pm 2.4$ & $29.7 \pm 5.8^{\text {bcd }}$ \\
Group B & $28.9 \pm 1.2$ & $31.0 \pm 1.6^{\mathrm{c}}$ & $24.0 \pm 1.2$ & $24.7 \pm 1.5^{\mathrm{e}}$ & $32.0 \pm 3.2$ & $37.6 \pm 4.6^{\mathrm{c}}$ & $20.0 \pm 2.3$ & $22.0 \pm 3.5^{\mathrm{e}}$ & $37.6 \pm 2.6$ & $41.6 \pm 2.6^{\text {ace }}$ \\
Group C & $30.9 \pm 1.6^{\text {ad }}$ & $33.4 \pm 1.1^{\text {abde }}$ & $25.3 \pm 1.7$ & $26.0 \pm 0.8^{\mathrm{d}}$ & $37.3 \pm 4.5$ & $45.0 \pm 3.8^{\text {abde }}$ & $23.1 \pm 3.9$ & $23.6 \pm 2.3^{\mathrm{d}}$ & $37.3 \pm 3.1$ & $46.9 \pm 2.7^{\mathrm{bde}}$ \\
Group D & $28.3 \pm 2.3^{\mathrm{c}}$ & $29.3 \pm 2.4^{\mathrm{c}}$ & $23.6 \pm 1.7$ & $23.7 \pm 2.0^{\mathrm{ce}}$ & $30.7 \pm 6.3$ & $33.4 \pm 6.7^{\mathrm{c}}$ & $19.3 \pm 3.8$ & $19.9 \pm 4.0^{\mathrm{ce}}$ & $36.8 \pm 3.6$ & $40.4 \pm 2.6^{\text {ace }}$ \\
Group E & $29.9 \pm 2.3^{\mathrm{a}}$ & $31.0 \pm 1.4^{\mathrm{c}}$ & $24.9 \pm 1.9$ & $26.6 \pm 1.0^{\text {bd }}$ & $34.9 \pm 6.2$ & $37.8 \pm 4.0^{\mathrm{c}}$ & $22.0 \pm 4.1$ & $26.1 \pm 2.5^{\text {bd }}$ & $36.9 \pm 3.1$ & $33.6 \pm 2.0^{\text {bcd }}$ \\
$F$ & 2.959 & 5.040 & 2.600 & 4.065 & 1.66 & 4.932 & 1.580 & 3.292 & 0.107 & 33.173 \\
$P$ & 0.036 & 0.003 & 0.056 & 0.009 & 0.162 & 0.004 & 0.205 & 0.024 & 0.979 & 0.000 \\
\hline
\end{tabular}

LVEF = left ventricular eject fraction, LVEDD = left ventricular end-diastolic dimension, LVESD = left ventricular end-systolic dimension, LVEDV $=$ left ventricular end-diastolic volume, and ESV $=$ left ventricular end-systolic volume. Values are means $\pm \mathrm{SD} .{ }^{\mathrm{a}} P<0.05$, compared with group A; ${ }^{\mathrm{b}} P<0.05$, compared with group $B$; ${ }^{\mathrm{c}} P<0.05$, compared with group $\mathrm{C}$; ${ }^{\mathrm{d}} P<0.05$, compared with group $\mathrm{D}$; and ${ }^{\mathrm{e}} P<0.05$, compared with group E.

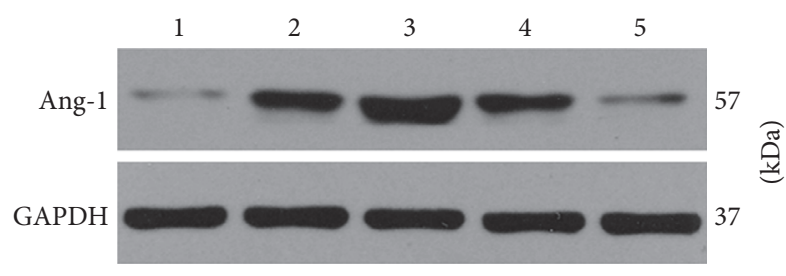

(a)

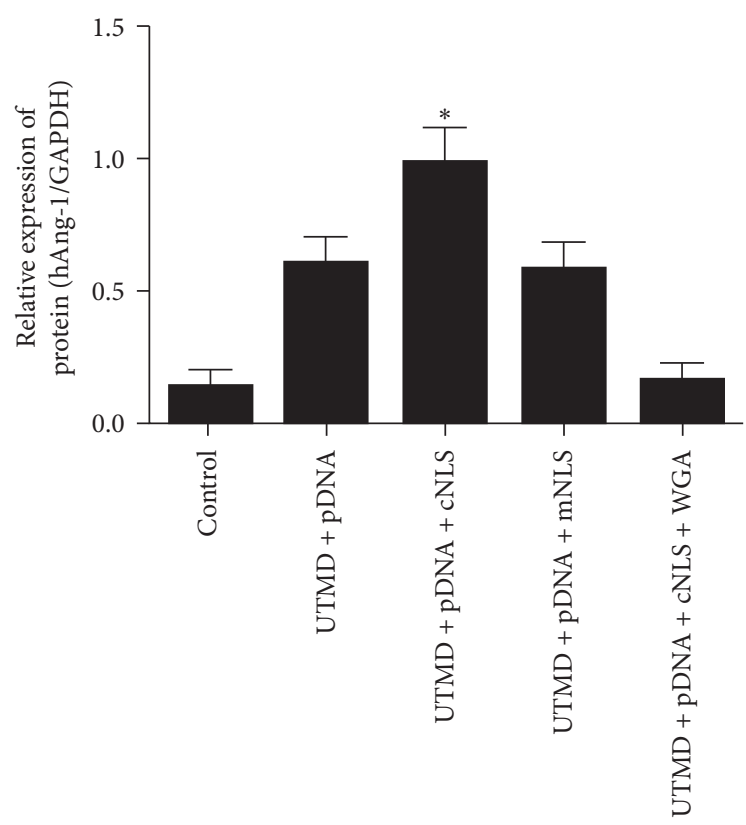

(b)

FIGURE 4: Western Blot analysis of Ang-1 protein expression in the MI border regions of canines. (a) Myocardial Ang-1 and GAPDH protein levels quantified using a Western Blot 3 days after UTMDmediated Ang-1 transfection. Lanes 1-5 represented groups A, B, C, D, and E, respectively. (b) Quantification of the Ang-1 protein relative expression levels was performed in the MI border regions of canines in all groups. The Ang-1 was normalized by GAPDH. The Ang-1 protein expression was the highest in group $C$; ${ }^{*} P<0.05$ compared to the other four groups.
4.6. Determination of cTnI. The change of the level of the serum cTnI showed the same trend in all five groups. The cTnI significantly increased at $24 \mathrm{hrs}$ after MI and gradually decreased. There was no significant difference in cTnI before and after gene transfection $(P>0.05)$, which indicated that this gene transfection system did not cause extra damage to cardiomyocytes compared to simple UTMD gene delivery (Table 2).

\section{Discussion}

Refractory ischemic heart disease is a severe disease with high mortality and morbidity and affects public health and living quality worldwide. Currently, UTMD-mediated gene delivery for neovessel formation is considered a promising nonviral system for treating myocardial ischemia $[18,19]$. In this study, we investigated the efficiency of UTMD combined with NLS for treating myocardial ischemia. The novelty of the study is that the UTMD-NLS gene delivery system allows gene transport with dual-targeting in terms of membrane and nuclear barriers and can achieve a better improvement of the angiogenesis and cardiac function than can UTMD transfection alone.

Three major factors have been reported to be responsible for the poor transfection efficiency of exogenous genes, including the cell membrane barrier, cytoplasmic degradation, and the nuclear membrane barrier [20]. To date, few studies have confirmed that ultrasound irradiation can release DNA into the cytoplasm and pass through the nuclear membrane, regardless of microbubble destruction, as long as the ultrasonic frequency is high enough and the total radiation energy is sufficient. However, this process has a long duration $(30 \mathrm{~min})$, which requires ultrasound irradiation, and the cell survival rate is lower than $80 \%$ [21]. In this study, a functional group (termed as NLS) was experimentally utilized in combination with the UTMD technique with the aim of improving the gene transferring efficiency instead of long-term ultrasound exposure.

NLS, which is a functional peptide derived from eukaryotic nucleoprotein and viral protein, could effectively mediate 

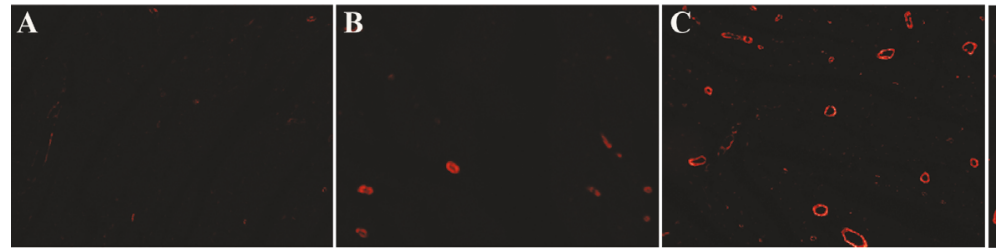

(a)

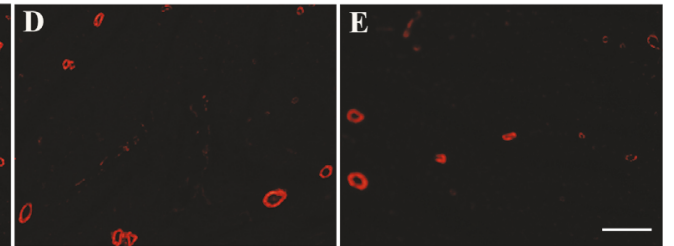

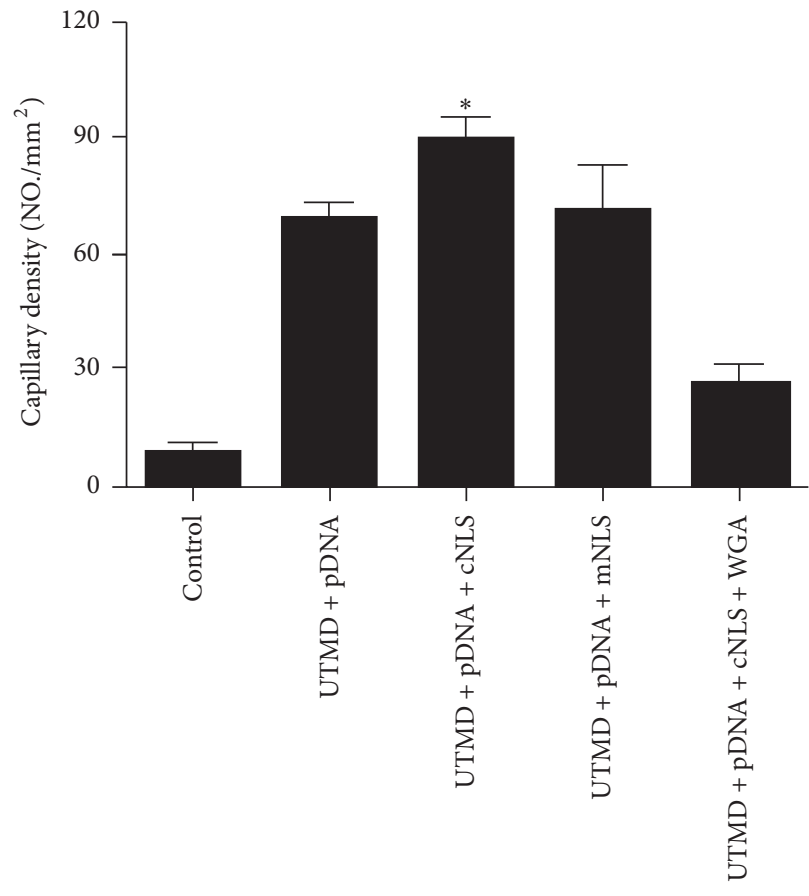

(b)

FIGURE 5: Angiogenesis after UTMD-mediated Ang-1 transfection in the MI border regions on day 28. (a) Representative images of capillary density from all groups on day 28 after transfection. The images were observed under a magnification of $200 x$. Scale bar $=20 \mu \mathrm{m}$. (b) The quantification of capillary density in group $\mathrm{C}$ was significantly higher compared to the other groups $\left({ }^{*} P<0.01\right)$.

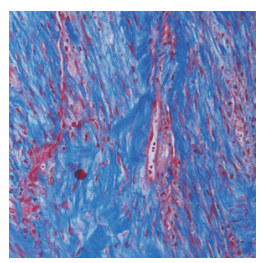

(a)

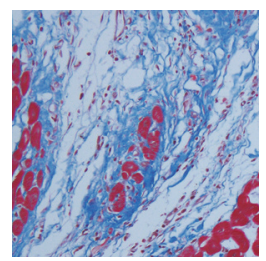

(b)

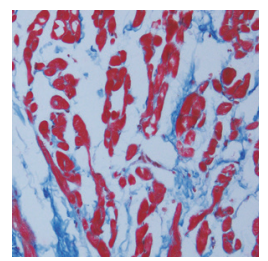

(c)

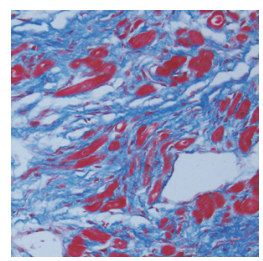

(d)

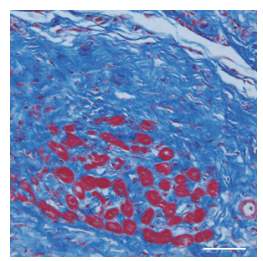

(e)

Figure 6: Masson trichrome staining of the infarcted zone on day 28 after Ang-1 gene transfection. Representative photomicrographs show the fibrosis of infarcted myocardium (myocardium in red, fibrosis in blue, magnification $100 \mathrm{x}$, scale bar $=100 \mu \mathrm{m}$ ). Compared with other groups, fibrosis of the infarct zone was obviously suppressed in group C, which was featured by less collagen fiber and formation of more normal myocardium. (a), (b), (c), (d), and (e) represented groups A, B, C, D, and E, respectively.

the intranuclear transport of molecules [22]. PKKKRKV, which is the minimum NLS of SV40T antigen, has been commonly used in the construction of nonviral genetic vector because it shows high transfection efficiency and low immunogenicity $[23,24]$. For example, Park et al. transfected the human lipocytes using PEI-NLS-GFP labeled plasmid, which showed higher gene transfer potency than the nonNLS group, even on day 7 [25]. At the same time, they reported that NLS contributed to the stability of exogenous DNA fragments by preventing the degradation of transferred genes in cytoplasm [26]. Jeon et al. transfected the human skin fibroblasts using poly (lactide-co-glycolide) (PLGA) 


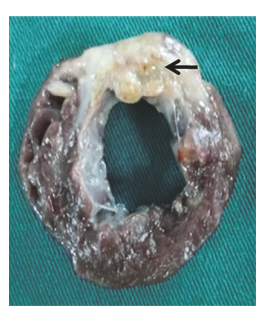

(a)

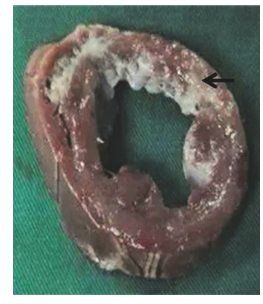

(b)

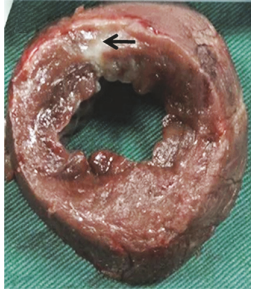

(c)

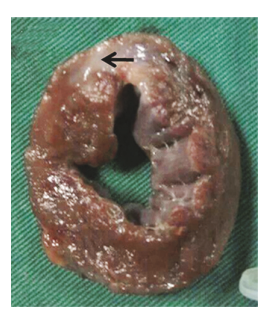

(d)

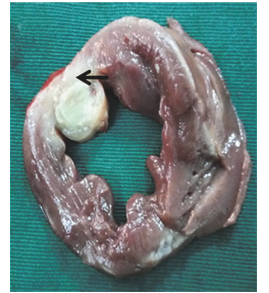

(e)

FIGURE 7: Pathomorphology of infarcted myocardium after Ang-1 gene transfection. Pathological specimens of the left ventricular short axis at the papillary muscle level from all groups on day 28 after Ang-1 transfection in canines. The tissue in white (marked by black arrow) indicated infarcted myocardial, and the tissue in red represented the noninfarcted myocardium. A wide range of tissue in white indicated more MI area in (a), whereas a higher percentage of tissue in red indicated noninfarcted myocardium in (c). (a), (b), (c), (d), and (e) represented groups A, $\mathrm{B}, \mathrm{C}, \mathrm{D}$, and $\mathrm{E}$, respectively.

TABLE 2: Serum cTnI at different time points in each group $(\bar{x} \pm s)$.

\begin{tabular}{|c|c|c|c|c|c|}
\hline Groups & pre-MI & MI-4h & MI-24h & Trans-D1 & Trans-D6 \\
\hline Group A & $49.6 \pm 4.4$ & $72.6 \pm 6.9$ & $147.4 \pm 6.8$ & $68.6 \pm 4.8$ & $53.6 \pm 3.8$ \\
\hline Group B & $53.0 \pm 5.4$ & $70.6 \pm 7.5$ & $152.0 \pm 5.6$ & $67.6 \pm 5.5$ & $54.4 \pm 4.6$ \\
\hline Group C & $56.6 \pm 5.9$ & $71.8 \pm 7.2$ & $153.8 \pm 3.4$ & $69.6 \pm 4.0$ & $56.0 \pm 5.4$ \\
\hline Group D & $56.4 \pm 5.9$ & $72.8 \pm 9.6$ & $153.6 \pm 7.3$ & $67.6 \pm 3.4$ & $55.6 \pm 7.4$ \\
\hline Group E & $56.6 \pm 5.3$ & $71.8 \pm 9.09$ & $158.8 \pm 7.2$ & $76.0 \pm 8.6$ & $58.4 \pm 4.3$ \\
\hline$F$ & 1.653 & 0.057 & 2.151 & 2.003 & 0.608 \\
\hline$P$ & 0.200 & 0.994 & 0.112 & 0.133 & 0.662 \\
\hline
\end{tabular}

Pre-MI = before myocardial infarction, MI- $4 \mathrm{~h}=4$ hours after myocardial infarction, MI-12 $\mathrm{h}=12$ hours after myocardial infarction, Trans-D1 $=1$ day after transfection, and Trans-D6 $=6$ days after transfection. Values are means \pm SD.

combined with NLS and plasmid DNA, which indicated that NLS conjugation enhanced the gene transfection efficiency by 1.2- 3.2-fold over 13 days in vitro, and the pDNA was released from PLGA nanospheres over 9 days [27]. In this study, a gene delivery system was constructed based on the biological function of UTMD and NLS. Our results showed that the transfection efficiency of the Ang-1 gene was approximately 1.6-fold compared to the UTMD system alone in the ischemic myocardium of dogs. Additionally, the number of neovessels formed in the MI region increased, along with reduced myocardial scarring and fibrosis. Moreover, the cardiac function was significantly improved. Based on this outcome, we considered that the system was nucleophilic and could target both the cell membrane and nuclear membrane. To further testify the roles of NLS in the delivery system, a mutational NLS was used. As shown previously, an accurate NLS sequence and structure are the premise for the transport of a receptor protein and nuclear pore complex. However, in the presence of mutation in ${ }^{126}$ PKKKRKV ${ }^{132}$, the linkage and amino acid sequences may change, which results in transport loss into the nucleus because the mutational NLS is not recognized by the receptor $[28,29]$. In addition, to clarify the nuclear transport of cNLS, WGA was used to block the nuclear pore, which then obstructed the binding of the NLS and the receptors. Although WGA did not affect the binding of plasmid DNA and NLS, the DNA could not be transported into the nucleus $[30,31]$. Our results showed that mNLS or WGA could decrease the Ang-1 transfection efficiency and protein expression. The expression of the Ang1 protein in group $\mathrm{C}$ was approximately 1.7 -fold that of group $\mathrm{D}$ and 6.2 -fold that of group E. This outcome indicated that dysfunction and/or blocking of NLS would significantly impair the delivery of therapeutic genes.

Concerning the gene delivery impact, the combination mode of NLS and plasmid DNA needs to be considered. NLS contained basic amino acids with positive charges under neutral conditions and could couple DNA with negative charges through electroadsorption. According to a previous study, the binding of NLS and DNA via electrostatic interaction could facilitate the entry of nonnuclear protein into the nucleus [32]. The disadvantage of this coupling mode was the possibility of the dissociation of NLS and DNA, which weakened the DNA in the nucleus. To strengthen the combination, the covalent binding mode was used in some studies, but special attention should be paid to prevent NLS from combining with functional gene expression components; otherwise, the gene cannot be well-expressed [33]. Based on this approach, we chose electrostatic binding in this study, which was easy to perform and could maintain the integrity of the NLS and DNA fragment [34].

Regarding potential applications, it is necessary to determine the safety while enhancing the efficiency of gene expression. The difference of $\mathrm{cTnI}$ was not significant on day 1 or day 6 after transfection among all groups, which suggested that NLS did not cause extra cardiomyocytes injury under UTMD. The cell injury during the gene delivery might be associated with the increased cell membrane permeability 
caused by cavitation effects of UTMD, whereas, in this study, it was depending on the function of NLS rather than on microbubbles or long-duration ultrasound exposure to help DNA enter the nucleus; thus the negative bio-effects on the cell should not be more severe than that observed when using UTMD alone.

There are some limitations to this study. Although NLS has been extensively utilized in molecular research, its roles in gene delivery into the nucleus are still not well defined. Therefore, we could only focus on the therapeutic impact without illustrating the potential mechanism. In addition, the transfection parameters still need to be explored further because we only optimized the molar ratio of NLS to plasmid $\left(10^{4}: 1\right)$ based on a previous description [35] and our previous data. More experiments are needed to determine the collocation among transfection parameters, such as ultrasound exposure (e.g., irradiation energy, density, and duration) and NLS concentration.

\section{Conclusions}

In conclusion, the nucleophilic delivery system constructed from the combination of UTMD and NLS contributed to the transfection efficiency by promoting a therapeutic gene in the nucleus and effectively increasing the angiogenesis in a canine myocardial infarction model.

\section{Conflicts of Interest}

The authors declare that they have no conflicts of interest.

\section{Authors' Contributions}

Jingjing Cui and Qing Deng equally contributed to this work.

\section{Acknowledgments}

This study was supported by Chinese National Science Foundation Committee Grant (nos. 81471674 and 81501495).

\section{References}

[1] R. M. Blaese, K. W. Culver, A. D. Miller et al., "T lymphocytedirected gene therapy for ADA- SCID: initial trial results after 4 years," Science, vol. 270, no. 5235, pp. 475-480, 1995.

[2] A. R. Carson, C. F. McTiernan, L. Lavery et al., "Ultrasoundtargeted microbubble destruction to deliver siRNA cancer therapy," Cancer Research, vol. 72, no. 23, pp. 6191-6199, 2012.

[3] D. Yang, K.-B. Tan, Y.-H. Gao, H. Liu, and W.-X. Yang, "Effects of diagnostic ultrasound-targeted microbubble destruction on permeability of normal liver in rats," Ultrasonics, vol. 52, no. 8, pp. 1065-1071, 2012.

[4] R. K. Schlicher, J. D. Hutcheson, H. Radhakrishna, R. P. Apkarian, and M. R. Prausnitz, "Changes in Cell Morphology Due to Plasma Membrane Wounding by Acoustic Cavitation," Ultrasound in Medicine \& Biology, vol. 36, no. 4, pp. 677-692, 2010.

[5] N. A. Geis, H. A. Katus, and R. Bekeredjian, "Microbubbles as a vehicle for gene and drug delivery: current clinical implications and future perspectives," Current Pharmaceutical Design, vol.18, no. 15, pp. 2166-2183, 2012.

[6] Z.-Y. Chen, F. Yang, Y. Lin et al., "New development and application of ultrasound targeted microbubble destruction in gene therapy and drug delivery," Current Gene Therapy, vol. 13, no. 4, pp. 250-274, 2013.

[7] V. Oberle, G. De Jong, J. I. Drayer, and D. Hoekstra, "Efficient transfer of chromosome-based DNA constructs into mammalian cells," Biochimica et Biophysica Acta-Gene Structure and Expression, vol. 1676, no. 3, pp. 223-230, 2004.

[8] J. D. Larsen, N. L. Ross, and M. Sullivan, "Requirements for the nuclear entry of polyplexes and nanoparticles during mitosis," The Journal of Gene Medicine, vol. 14, no. 9-10, pp. 580-589, 2012.

[9] F. Labat-Moleur, A.-M. Steffan, C. Brisson et al., "An electron microscopy study into the mechanism of gene transfer with lipopolyamines," Gene Therapy, vol. 3, no. 11, pp. 1010-1017, 1996.

[10] C. R. Mayer, N. A. Geis, H. A. Katus, and R. Bekeredjian, "Ultrasound targeted microbubble destruction for drug and gene delivery," Expert Opinion on Drug Delivery, vol. 5, no. 10, pp. 1121-1138, 2008.

[11] H. Chen and J. H. Hwang, "Ultrasound-targeted microbubble destruction for chemotherapeutic drug delivery to solid tumors," Journal of Therapeutic Ultrasound, vol. 1, no. 1, article no. 10, 2013.

[12] P. Opanasopit, T. Rojanarata, A. Apirakaramwong, T. Ngawhirunpat, and U. Ruktanonchai, "Nuclear localization signal peptides enhance transfection efficiency of chitosan/ DNA complexes," International Journal of Pharmaceutics, vol. 382, no. 1-2, pp. 291-295, 2009.

[13] T. Kawazu, H. Kanzaki, A. Uno, H. Azuma, and T. Nagasaki, "HVJ-E/importin- $\beta$ hybrid vector for overcoming cytoplasmic and nuclear membranes as double barrier for non-viral gene delivery," Biomedicine \& Pharmacotherapy, vol. 66, no. 7, pp. 519-524, 2012.

[14] M. Duvshani-Eshet, L. Baruch, E. Kesselman, E. Shimoni, and M. Machluf, "Therapeutic ultrasound-mediated DNA to cell and nucleus: Bioeffects revealed by confocal and atomic force microscopy," Gene Therapy, vol. 13, no. 2, pp. 163-172, 2006.

[15] S. Ibaragi, N. Yoshioka, H. Kishikawa et al., "Angiogeninstimulated rRNA transcription is essential for initiation and survival of AKT-induced prostate intraepithelial neoplasia," Molecular Cancer Research, vol. 7, no. 3, pp. 415-424, 2009.

[16] S. Cao, Q. Zhou, J. Chen et al., "Enhanced effect of nuclear localization signal peptide during ultrasound-targeted microbubble destruction-mediated gene transfection," Molecular Medicine Reports, vol. 16, no. 1, pp. 565-572, 2017.

[17] J. S. Yuan, A. Reed, F. Chen, and C. N. Stewart Jr., "Statistical analysis of real-time PCR data," BMC Bioinformatics, vol. 7, pp. 563-569, 2006.

[18] R. F. J. Kwekkeboom, J. P. G. Sluijter, B. J. Van Middelaar et al., "Increased local delivery of antagomir therapeutics to the rodent myocardium using ultrasound and microbubbles," Journal of Controlled Release, vol. 222, pp. 18-31, 2016.

[19] M. B. Preda and G. Valen, "Evaluation of gene and cell-based therapies for cardiac regeneration," Current Stem Cell Research \& Therapy, vol. 8, no. 4, pp. 304-312, 2013.

[20] F. Sadeghian, S. Hosseinkhani, A. Alizadeh, and A. Hatefi, "Design, engineering and preparation of a multi-domain fusion vector for gene delivery," International Journal of Pharmaceutics, vol. 427, no. 2, pp. 393-399, 2012. 
[21] M. Duvshani-Eshet and M. Machluf, "Therapeutic ultrasound optimization for gene delivery: A key factor achieving nuclear DNA localization," Journal of Controlled Release, vol. 108, no. 23, pp. 513-528, 2005.

[22] M. Marfori, A. Mynott, J. J. Ellis et al., "Molecular basis for specificity of nuclear import and prediction of nuclear localization," Biochimica et Biophysica Acta (BBA) - Molecular Cell Research, vol. 1813, no. 9, pp. 1562-1577, 2011.

[23] R. Schirmbeck, S. A. König-Merediz, P. Riedl et al., "Priming of immune responses to hepatitis B surface antigen with minimal DNA expression constructs modified with a nuclear localization signal peptide," Journal of Molecular Medicine, vol. 79, no. 5-6, pp. 343-350, 2001.

[24] C. Zheng, C. Juhls, D. Oswald et al., "Effect of different nuclear localization sequences on the immune responses induced by a MIDGE vector encoding bovine herpesvirus-1 glycoprotein D," Vaccine, vol. 24, no. 21, pp. 4625-4629, 2006.

[25] E. Park, H.-B. Cho, and K. Takimoto, "Effective gene delivery into adipose-derived stem cells: Transfection of cells in suspension with the use of a nuclear localization signal peptideconjugated polyethylenimine," Cytotherapy, vol. 17, no. 5, pp. 536-542, 2015.

[26] B.-K. Kim, H. Kang, K.-O. Doh et al., "Homodimeric SV40 NLS peptide formed by disulfide bond as enhancer for gene delivery," Bioorganic \& Medicinal Chemistry Letters, vol. 22, no. 17, pp. 5415-5418, 2012.

[27] O. Jeon, H.-W. Lim, M. Lee, S. J. Song, and B.-S. Kim, "Poly(1lactide-co-glycolide) nanospheres conjugated with a nuclear localization signal for delivery of plasmid DNA," Journal of Drug Targeting, vol. 15, no. 3, pp. 190-198, 2007.

[28] C. Bernis and D. J. Forbes, "Analysis of nuclear reconstitution, nuclear envelope assembly, and nuclear pore assembly using Xenopus in vitro assays," Methods in Cell Biology, vol. 122, pp. 165-191, 2007.

[29] S. A. Adam, T. J. Lobl, M. A. Mitchell, and L. Gerace, "Identification of specific binding proteins for a nuclear location sequence," Nature, vol. 337, no. 6204, pp. 276-279, 1989.

[30] J. Kim, A. Izadyar, N. Nioradze, and S. Amemiya, "Nanoscale mechanism of molecular transport through the nuclear pore complex as studied by scanning electrochemical microscopy," Journal of the American Chemical Society, vol. 135, no. 6, pp. 2321-2329, 2013.

[31] W. Yang, "Distinct, but not completely separate spatial transport routes in the nuclear pore complex," Nucleus, vol. 4, no. 3, pp. 166-175, 2013.

[32] N. E. Bernardes, A. A. S. Takeda, T. R. Dreyer, F. Z. Freitas, M. C. Bertolini, and M. R. M. Fontes, "Structure of importin$\alpha$ from a filamentous fungus in complex with a classical nuclear localization signal," PLOS ONE, vol. 10, no. 6, p. e0128687, 2015.

[33] K. H. Bremner, L. W. Seymour, A. Logan, and M. L. Read, "Factors influencing the ability of nuclear localization sequence peptides to enhance nonviral gene delivery ," Bioconjugate Chemistry, vol. 15, no. 1, pp. 152-161, 2004.

[34] V. Escriou, M. Carrière, D. Scherman, and P. Wils, "NLS bioconjugates for targeting therapeutic genes to the nucleus," Advanced Drug Delivery Reviews, vol. 55, no. 2, pp. 295-306, 2003.

[35] M. Duvshani-Eshet, H. Keren, S. Oz, I. S. Radzishevsky, A. Mor, and M. Machluf, "Effect of peptides bearing nuclear localization signals on therapeutic ultrasound mediated gene delivery," The Journal of Gene Medicine, vol. 10, no. 10, pp. 1150-1159, 2008. 

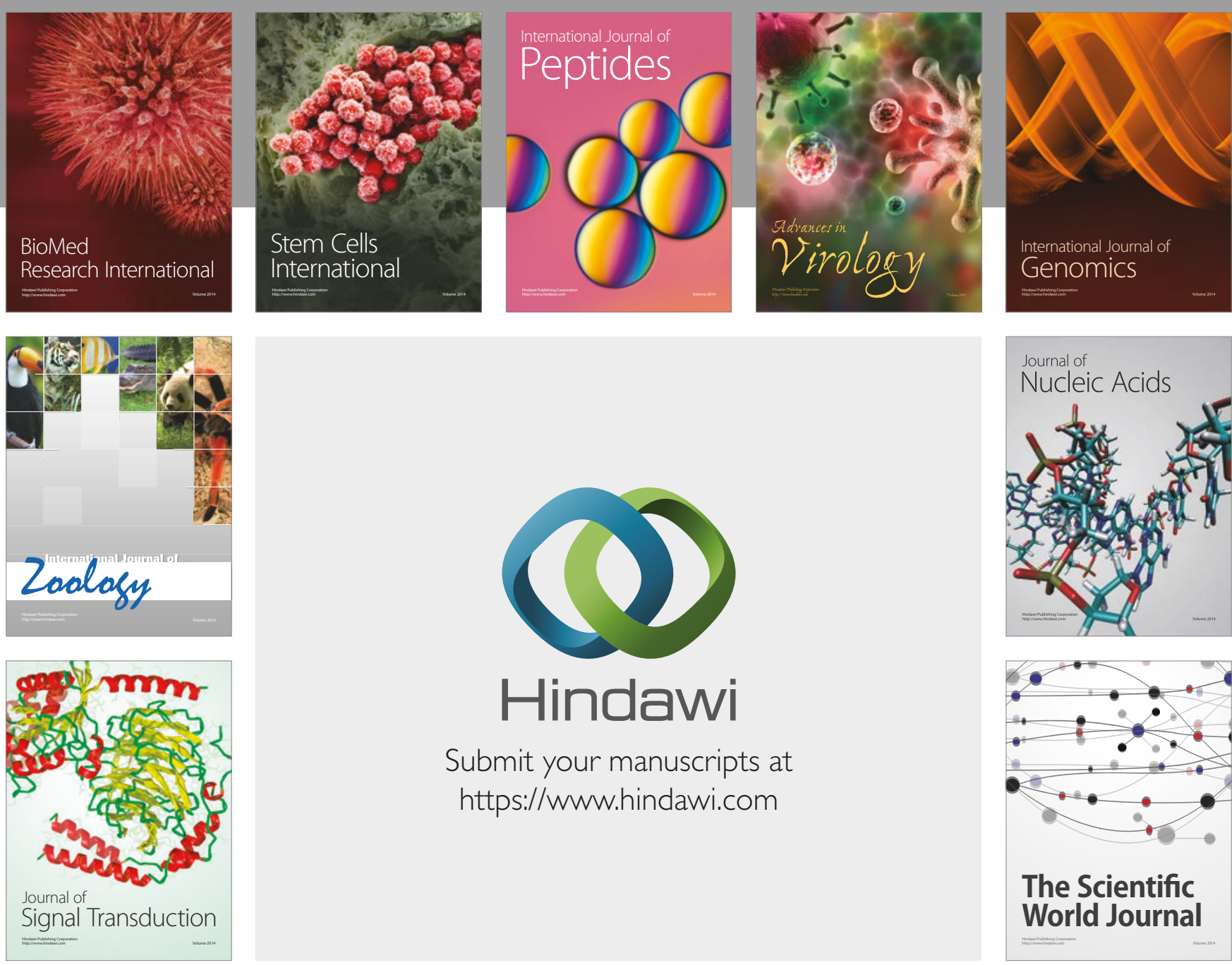

Submit your manuscripts at

https://www.hindawi.com
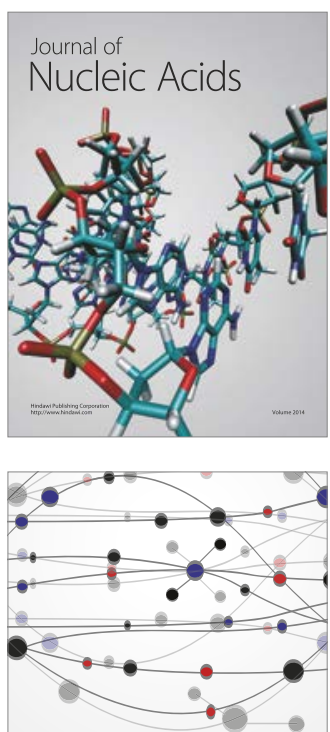

The Scientific World Journal

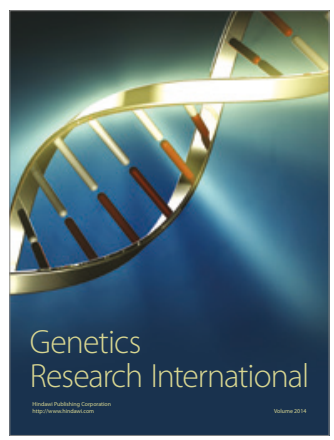

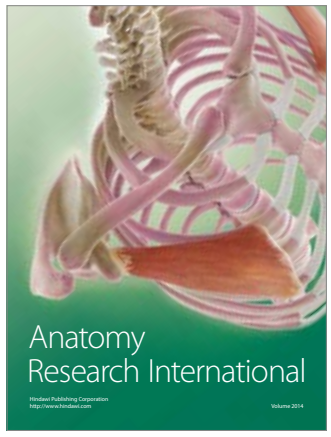

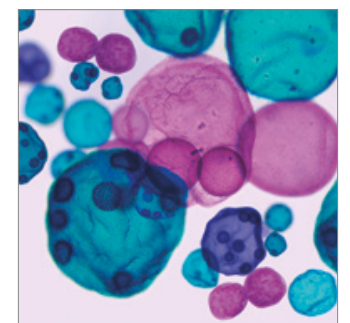

International Journal of Microbiology
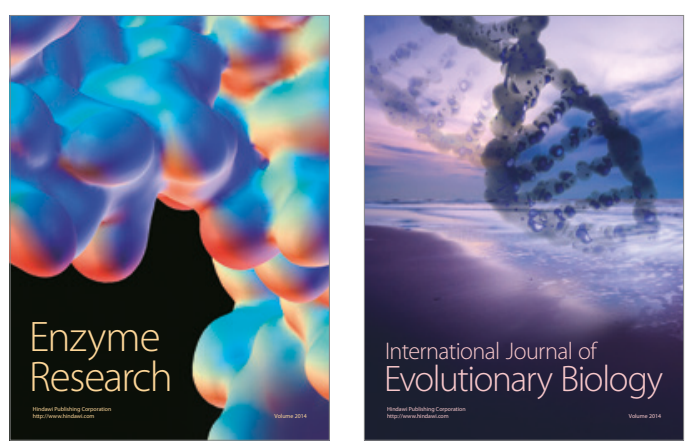
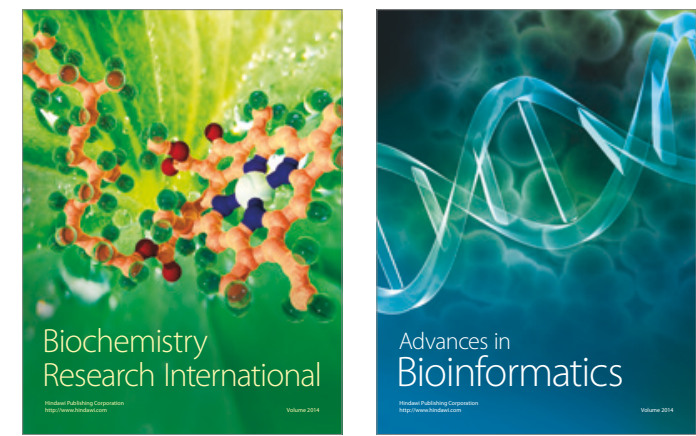

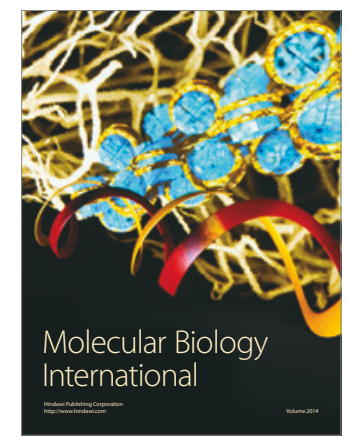

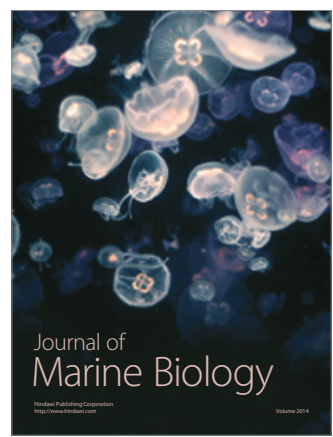

